ITC $3 / 49$

Information Technology and Control

Vol. 49 / No. 3 / 2020

pp. 320-334

DOI 10.5755/j01.itc.49.3.2547\%
A WIP Vehicle Control Method Based on Improved Artificial Potential Field Subject to Multi-Obstacle Environment

Received 2020/03/11

Accepted after revision 2020/05/30

HOW TO CITE: Ning, Y., Yue, M., Guo, L., Zhao, J. (2020). A WIP Vehicle Control Method Based on Improved Artificial Potential Field Subject to Multi-Obstacle Environment. Information Technology and Control, 49(3), 320-334. https://doi.org/10.5755/j01.itc.49.3.25477

\title{
A WIP Vehicle Control Method Based on Improved Artificial Potential Field Subject to Multi-Obstacle Environment
}

\section{Yigao Ning}

School of Automotive Engineering, Dalian University of Technology, Dalian 116024, China

\section{Ming Yue*}

School of Automotive Engineering, Dalian University of Technology, Dalian 116024, China; State Key Laboratory of Robotics and System, Harbin Institute of Technology, Harbin 150001, China

\section{Lie Guo, Jian Zhao}

School of Automotive Engineering, Dalian University of Technology, Dalian 116024, China

*Corresponding author: yueming@dlut.edu.cn

This paper presents a control method for a WIP vehicle in multi-obstacle environment based on the improved artificial potential field. Firstly, an improved artificial potential field (IAPF) is developed, where a safe distance is introduced to the existing repulsive potential field to solve the security issue, while the probable local minima caused by the obstacle around the target can also be eliminated simultaneously. Next, an obstacle avoidance controller is designed based on the IAPF, where the nonholonomic constraint and underactuated characteristic of the WIP vehicle are fully considered, and the stability condition of the system is analyzed by means of the related control theory. Moreover, to further improve the control performance, a key parameter that play an important role in the controller is adjusted by taking advantage of fuzzy logic, and detailed analyses are given to demonstrate its necessity and effectiveness. Finally, considering a motion environment that contains dense obstacles, narrow corridor and an obstacle near the target, numerical simulations are conducted to validate the proposed method, whose results indicate that the method has a good performance to control the WIP vehicle in multi-obstacle environment. 
KEYWORDS: Wheeled inverted pendulum (WIP) vehicle, nonholonomic constraint, underactuated characteristic, improved artificial potential field (IAPF), fuzzy logic, multi-obstacle environment.

\section{Introduction}

Wheeled inverted pendulum (WIP) vehicles, as a typical mobile robots, have become widely available and continuously attracted much attentions in recent decades, owing to their compact construction, high maneuverability, and low energy consumption characteristics [3, 7, 15, 33, 38]. In the practical application process, such as home service, industrial production, transportation, space probe, etc., the WIP vehicle will inevitably encounter various obstacles, which seriously influence the implementation of related tasks $[2,32,40]$. Moreover, the WIP vehicle subjects to the nonholonomic constraint between the wheels and ground due to the pure rolling motion [6, 11, 21, 34], and it is also a typical underactuated system that the number of control inputs is less than the degrees of freedom $[8,14,29,37]$, both of which make the control of the vehicle more difficult. Therefore, developing a control method for the WIP vehicle in multi-obstacle environment not only has important theoretical meaning, but also has great practical significance.

To realize the better control performance of the WIP vehicle, plenty of valuable results have been carried out by many researchers. For example, dynamic surface control [13] and nonlinear optimal control [17] are proposed to balance the vehicle body; terminal sliding mode control [11] and energy shaping [35] are developed to realize the forward motion control of the WIP vehicle; neural-adaptive output control [20] and robust model predictive control [36] are presented to achieve the trajectory tracking. Specifically, a suitable coordinates transformation is introduced in [5] to formulate the WIP model into a parametric strict feedback form based on the nonlinear model, and then an adaptive backstepping control method is applied to achieve output tracking of the WIP vehicle. In addition, to achieve fast positioning and maintain the balance of robot body simultaneously, Liang et al. [22] propose a time-optimal trajectory planning-based method for WIP robots on an inclined plane, where system state variables and their derivatives can be ensured in given ranges. Nonetheless, it is important to note that these methods are mainly focused on the control of WIP vehicles in barrier-free environment without consideration given to the vehicle control in obstacle environment. Furthermore, to avoid a single obstacle, a trajectory planning and tracking control scheme is proposed for the WIP vehicle in [27], by which the vehicle can get around the obstacle with a constant velocity and return to the established route ultimately. However, although the above studies have done much effort to the WIP vehicle control from several aspects, the control in multi-obstacle environment is not involved and is still a challenging problem.

For the general mobile robots control in a complex multi-obstacle environment, there are also abundant approaches presented in literature, such as potential field approach [4], elastic band approach [10], and dynamic window approach [28], etc. Especially, Khatib [16] proposes an artificial potential field (APF) approach, which has outstanding advantages of simplicity, high-efficiency and smooth trajectory generation and has been widely used in obstacles avoidance of robots. Afterwards, to deal with the problem of goals nonreachable with obstacles nearby in traditional APF, new potential functions for mobile robots are proposed by taking the relative distance between the robot and the goal into consideration in [9]. To solve the potential field local minimum problem, Mabrouk et al. [23] propose a new extended APF by using dynamic internal agent states, which has been demonstrated in problems with a single local minimum and a maze with multiple local minima. Furthermore, Sun et al. [30] propose an optimized APF algorithm for unmanned aerial vehicle systems, which has been validated in quantitative test simulation models on Matlab platform. Nevertheless, the robots in most of these studies are regarded as mass points and can generate acceleration along arbitrary direction, which are not satisfied by a practical WIP vehicle. Therefore, the above methods cannot complete the effective control of the WIP vehicle in multi-obstacle environment, which motivates this study.

Enlightened by the aforementioned literature, this paper proposes a control method based on improved 
artificial potential field (IAPF) for the WIP vehicle in multi-obstacle environment. The main works and innovations are as follows: (1) a safe distance is introduced to the traditional repulsive potential field to develop IAPF, by which the security issue can be effectively solved; (2) the obstacle avoidance controller for the WIP vehicle is designed based on IAPF, where the nonholonomic and underactuated characteristics are fully considered, and the stability condition of the system is analyzed in detail; (3) an adjustment approach based on fuzzy logic is proposed for a key parameter that plays an important role in the obstacles avoidance controller, hence the stability and efficiency of the control method are both concerned.

The rest of the paper is organized as follows. The problem statement is introduced in Section 2, and the IAPF is presented in Section 3. Then the controller for obstacles avoidance of the WIP vehicle is designed in Section 4. Next, an adjustment approach based on fuzzy logic for a key parameter in the controller is proposed in Section 5. After that, numerical simulations that consider multiple types of obstacles are conducted in Section 6 to verify the effectiveness of the proposed method. Finally, some valuable conclusions are given in Section $\%$.

\section{Problem Statement}

The WIP vehicle is mainly composed of chassis, wheels, vehicle body, and other components [38], which can be described by the generalized coordinate vector $\boldsymbol{q}=[x, y, \varphi, \theta]^{\mathrm{T}}$, where $x, y, \varphi$, and $\theta$ represent the horizontal axis, vertical axis, steering angle, and tilt angle of vehicle body, respectively. Assume that there exists no slip between the wheels and the ground, and the pure rolling condition holds throughout, thus the vehicle satisfies the following nonholonomic constraint:

$$
\dot{x} \sin \varphi-\dot{y} \cos \varphi=0 \text {. }
$$

Remark 1. The above constraint means the WIP vehicle can only move along the tangential direction of the present motion trajectory at any moment, and cannot generate acceleration along arbitrary direction, which is one reason that the common APF approach is unable to be applied in the control of WIP vehicles directly.

Moreover, the dynamic model of the WIP vehicle can be formulated by Lagrangian modeling method, which can be further simplified based on the fact that $\theta$ remains in a small region, that is $\cos \theta \approx 1, \sin \theta \approx \theta$, and $\dot{\theta}^{2}=0$, as follows:

$$
\frac{2 J_{1} r}{d} \ddot{\varphi}=\tau_{\omega},
$$

$$
\frac{\left(4 m_{v} J_{2}-m_{b}^{2} L^{2}\right) r}{2 J_{2}} \ddot{x}_{v}+\frac{m_{b}^{2} g L^{2} r}{2 J_{2}} \theta=\tau_{v},
$$

$\frac{1}{m_{b} L} \ddot{\theta}+\frac{1}{2 J_{2}} \ddot{x}_{v}-\frac{g}{2 J_{2}} \theta=0$,

where $\quad \tau_{\omega}=\tau_{\mathrm{r}}-\tau_{1}, \quad \tau_{\mathrm{v}}=\tau_{\mathrm{r}}+\tau_{1}, \quad m_{\mathrm{v}}=I_{2} / r^{2}+m_{\mathrm{w}}+m_{\mathrm{c}} / 2+m_{\mathrm{b}} / 2$, $J_{1}=I_{1}+I_{3} / 2+I_{5} / 2+\left(m_{\mathrm{w}}+I_{2} / r^{2}\right) d^{2} / 4, \quad J_{2}=I_{4} / 2+I_{6} / 2+m_{\mathrm{b}} L^{2} /$ 2, $\dot{x}_{v}=\dot{x} \cos \varphi+\dot{y} \sin \varphi$, and $\tau_{l}$ and $\tau_{r}$ are the control torques for the left and right wheels, respectively. More details about the derivation procedures of this model, the system dynamics, and related parameters, etc. can be found in $[27,38]$ and references therein, which are omitted here for simplicity.

Remark 2. It can be seen from Equations (2) - (4) that there are only two control inputs $\tau_{\omega}$ and $\tau_{v}$, but three motion freedoms $\varphi, x_{\mathrm{v}}$ and $\theta$. Specifically, the longitudinal motion freedom $x_{\mathrm{v}}$ should be controlled by $\tau_{\mathrm{v}}$ while the regulation of the tilt angle of vehicle body $\theta$ also depends on $\tau_{v}$. This underactuated characteristic is the other reason that the common APF approach cannot be applied in the control of WIP vehicles directly.

Therefore, assume the posture of the vehicle and the information of obstacles around the WIP vehicle can be obtained by related sensors, then the control problem of the vehicle in multi-obstacle environment can be described as follows: given an initial posture $\boldsymbol{q}_{0}=\left[x_{0}\right.$, $\left.y_{0}, \varphi_{0}, \theta_{0}\right]^{\mathrm{T}}$, find proper control inputs $\tau_{\omega}$ and $\tau_{\mathrm{v}}$ to make the vehicle reach the target posture $\boldsymbol{q}_{\mathrm{f}}=\left[x_{\mathrm{f}}, y_{\mathrm{f}}, \varphi_{\mathrm{f}}, \theta_{\mathrm{f}}\right]^{\mathrm{T}}$ successfully, and at the same time avoid the obstacles in the environment without any collision. To solve the problem, this study intends to present a WIP vehicle control method based on improved artificial potential field, as shown in Figure 1, which will be developed in the following. 
Figure 1

Integrated framework

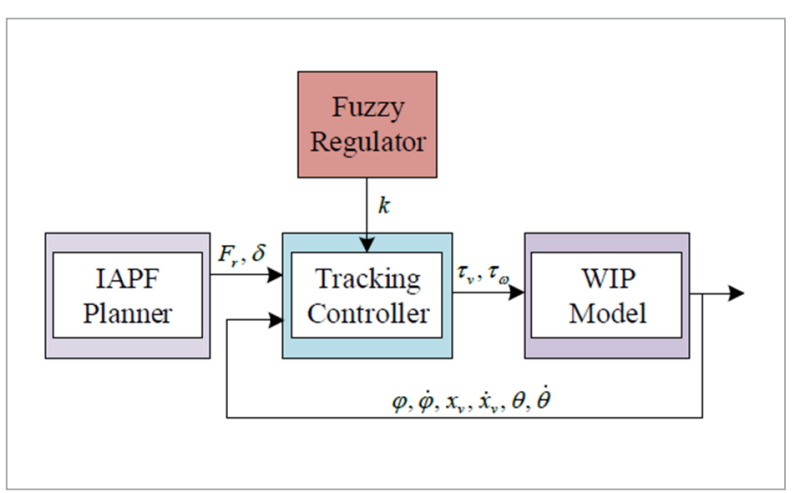

\section{Improved Artificial Potential Field}

The APF approach is widely used in robot collision avoidance because of its mathematical elegance, simple structure, and smooth motion trajectory, and some practical potential fields have been presented in literature [24-26]. Specifically, a commonly used attractive potential and a new repulsive potential function are adopted in [9] to overcome the problem of goals nonreachable with obstacles nearby (GNRON), which can make the global minimum be at the target point and eliminate the problem of local minima simultaneously [30], as follows:

$$
\begin{aligned}
& U_{t}(\boldsymbol{p})=\frac{1}{2} k_{a} \rho^{2}\left(\boldsymbol{p}, \boldsymbol{p}_{t}\right), \\
& U_{o}(\boldsymbol{p})=\left\{\begin{array}{lr}
\frac{1}{2} k_{r}\left(\frac{1}{\rho\left(\boldsymbol{p}, \boldsymbol{p}_{o}\right)}-\frac{1}{R_{o}}\right)^{2} \rho^{\varepsilon}\left(\boldsymbol{p}, \boldsymbol{p}_{t}\right), \\
0\left(\boldsymbol{p}, \boldsymbol{p}_{o}\right) \leq R_{o} \\
0, & \rho\left(\boldsymbol{p}, \boldsymbol{p}_{o}\right)>R_{o}
\end{array}\right.
\end{aligned}
$$

where $U_{t}(\boldsymbol{p})$ and $U_{\mathrm{o}}(\boldsymbol{p})$ denote the attractive potential field generated by the target point and the repulsive potential field generated by an obstacle, respectively; $k_{\mathrm{a}} \in R^{+}$and $k_{\mathrm{r}} \in R^{+}$represent the attractive potential field gain coefficient and repulsive potential field gain coefficient, respectively; $\boldsymbol{p}=(x, y), \boldsymbol{p}_{\mathrm{o}}=\left(x_{0}, y_{\mathrm{o}}\right)$ and $\boldsymbol{p}_{\mathrm{t}}=\left(x_{\mathrm{f}}, y_{\mathrm{f}}\right)$ represent the position of the robot, obstacle and target point, respectively; $R_{\mathrm{o}}$ represents the influence distance of the repulsive potential field; $\rho\left(\boldsymbol{p}, \boldsymbol{p}_{\mathrm{t}}\right)$ represents the distance between the vehicle and the target point; $\rho\left(\boldsymbol{p}, \boldsymbol{p}_{\mathrm{o}}\right)$ denotes the minimal distance between the vehicle and the obstacle; $\varepsilon$ is a design parameter that satisfies $\varepsilon \geq 1$ to guarantee the differentiability of $U_{0}(\boldsymbol{p})$. However, the WIP vehicle in reality is not a mass point but has a specific size, and the control delay is always existed, which may cause a security issue when applying the potential field (6) for obstacles avoidance control.

Aiming at this problem, a safe distance is introduced to the repulsive potential field to further improve its application performance in this study, and the IAPF is as follows:

$$
U_{o}(\boldsymbol{p})=\left\{\begin{array}{c}
\frac{1}{2} k_{r}\left(\frac{1}{\rho\left(\boldsymbol{p}, \boldsymbol{p}_{o}\right)-\sigma}-\frac{1}{R_{o}-\sigma}\right)^{2} \rho^{\varepsilon}\left(\boldsymbol{p}, \boldsymbol{p}_{t}\right), \\
\rho\left(\boldsymbol{p}, \boldsymbol{p}_{o}\right) \leq R_{o} \\
\rho\left(\boldsymbol{p}, \boldsymbol{p}_{o}\right)>R_{o}
\end{array}, \quad(7)\right.
$$

where $\sigma \in R^{+}$can ensure that the repulsive potential is sufficiently large when the vehicle confronts the obstacle at a safe distance, and guarantee the continuous change of the repulsive potential from zero to large enough in its coverage. In addition, it can be deduced from (5) and (7) that the total potential $U_{\text {total }}(\boldsymbol{p})=U_{\mathrm{t}}(\boldsymbol{p})+U_{\mathrm{o}}(\boldsymbol{p})$ arrives at its global minimum, namely zero, if and only if the vehicle reaches the target point, i.e., $\boldsymbol{p}=\boldsymbol{p}_{\mathrm{t}}$. Nevertheless, if $k_{\mathrm{a}}$ and $k_{\mathrm{r}}$ are not selected appropriately for a given $\varepsilon$, local minima may be existed, which may lead the vehicle to be trapped at these points and cannot reach the target point. For example, given $\boldsymbol{p}_{\mathrm{t}}=(2,1.5), \sigma=0.1, R_{\mathrm{o}}=2$, and choose $k_{\mathrm{a}}=2, k_{\mathrm{r}}=100, \varepsilon=3$, then it can be calculated that there is a local minimum at the point $(3.09,2.04)$, and the total potential is shown in Figure 2, where the obstacle is a circle of radius $r_{\mathrm{o}}=0.5$ with $\boldsymbol{p}_{\mathrm{o}}=(1,1)$. Furthermore, it can be found that the local minimum is on the line passing through the target point and the nearest boundary point on the obstacle, as shown in Figure 3 with the red dash-dot line, and this phenomenon is not an accident, which will be explained later in this study. Thus, to eliminate the local minimum, parameters $k_{\mathrm{a}}$ and $k_{\mathrm{r}}$ should be properly chosen for a concrete value of $\varepsilon$, and the choosing method is introduced in the following. 
Figure 2

Total potential with a local minimum

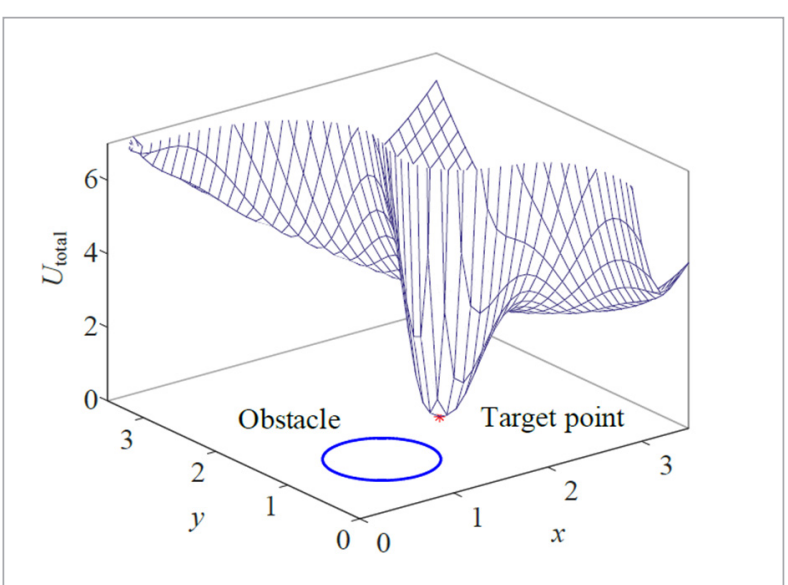

Figure 3

Total potential on the line with a local minimum

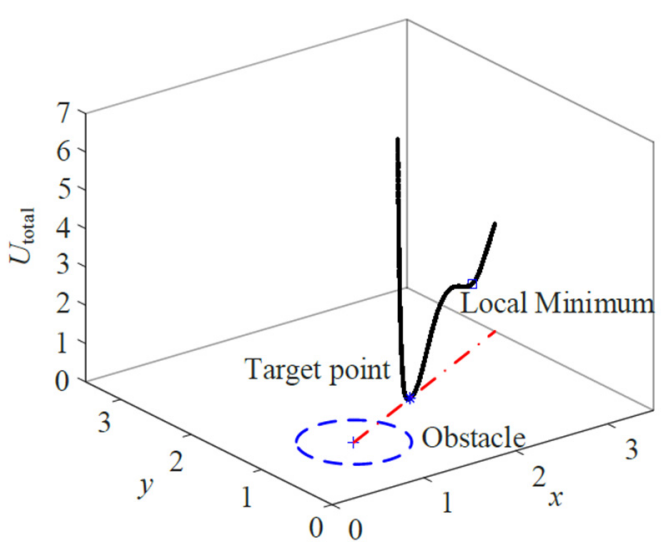

The WIP vehicle is propelled both by the attractive force from the target point and the repulsive force from the obstacle when the vehicle is within the influence area of the obstacle near to the target point, which can be obtained by calculating the negative gradient of the corresponding potential field (5) and ( 7 ), respectively, as follows:

$$
\begin{gathered}
\boldsymbol{F}_{t}(\boldsymbol{p})=-\nabla U_{t}(\boldsymbol{p})=k_{a} \rho\left(\boldsymbol{p}, \boldsymbol{p}_{t}\right)\left(-\nabla \rho\left(\boldsymbol{p}, \boldsymbol{p}_{t}\right)\right), \\
\boldsymbol{F}_{o}(\boldsymbol{p})=-\nabla U_{o}(\boldsymbol{p})= \\
\begin{cases}F_{r 1} \nabla \rho\left(\boldsymbol{p}, \boldsymbol{p}_{o}\right)+F_{r 2}\left(-\nabla \rho\left(\boldsymbol{p}, \boldsymbol{p}_{t}\right)\right), & \rho\left(\boldsymbol{p}, \boldsymbol{p}_{o}\right) \leq R_{o}, \\
0, & \rho\left(\boldsymbol{p}, \boldsymbol{p}_{o}\right)>R_{o}\end{cases}
\end{gathered}
$$

where

$$
F_{r 1}=k_{r}\left(\frac{1}{\rho\left(\boldsymbol{p}, \boldsymbol{p}_{o}\right)-\sigma}-\frac{1}{R_{o}-\sigma}\right) \frac{\rho^{\varepsilon}\left(\boldsymbol{p}, \boldsymbol{p}_{t}\right)}{\left(\rho\left(\boldsymbol{p}, \boldsymbol{p}_{o}\right)-\sigma\right)^{2}}
$$

$$
F_{r 2}=\frac{k_{r} \varepsilon}{2}\left(\frac{1}{\rho\left(\boldsymbol{p}, \boldsymbol{p}_{o}\right)-\sigma}-\frac{1}{R_{o}-\sigma}\right)^{2} \rho^{\varepsilon-1}\left(\boldsymbol{p}, \boldsymbol{p}_{t}\right),
$$

with $\nabla \rho\left(\boldsymbol{p}, \boldsymbol{p}_{\mathrm{o}}\right)$ and $-\nabla \rho\left(\boldsymbol{p}, \boldsymbol{p}_{\mathrm{t}}\right)$ being two unit vectors pointing from the obstacle to the robot and from the robot to the target point, respectively. As shown in Figure $4, F_{\text {total }}$ denotes the resultant of the repulsive force and attractive force; the component force $F_{\mathrm{r} 1}$ of the re-

\section{Figure 4}

Force condition of the WIP vehicle

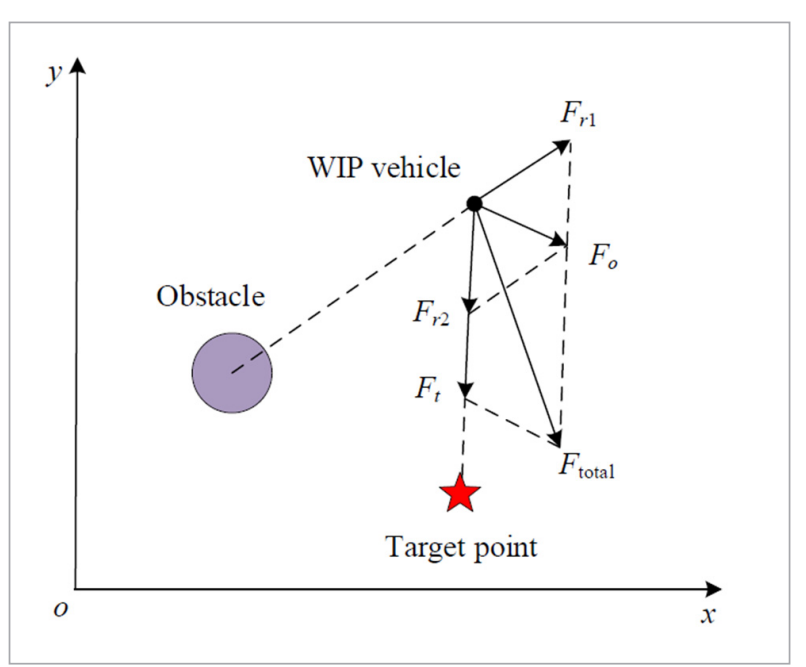

pulsive force $F_{\mathrm{o}}$ repulses the robot away from the obstacle; the other component force $F_{\mathrm{r} 2}$ attracts the robot toward the target point, which is in the same direction as the attractive force $F_{\mathrm{t}}$. Therefore, if the vehicle, the target point and the obstacle are not in collinear positions, there will be a nonzero force that actuates the vehicle to move toward this line. This means the local minima cannot exist outside the line that connects the target point and the obstacle, which only may appear on this line. In addition, the local minima in the situation that the obstacle is between the vehicle and the target point is not researched in this study, which may be solved by wall following method [9]. According to the analyses above and for convenience, we will focus 
on the case where the WIP vehicle, the target point, and the obstacle are collinear, with the vehicle and the obstacle locating at different sides of the target point. As shown in Figure 5, $z$ is the constant distance between the target point and the obstacle nearby, which satisfies $z=\rho\left(\boldsymbol{p}, \boldsymbol{p}_{\mathrm{o}}\right)-\rho\left(\boldsymbol{p}, \boldsymbol{p}_{\mathrm{t}}\right)>\sigma$.

Figure 5

Position relationship for the vehicle, target and obstacle

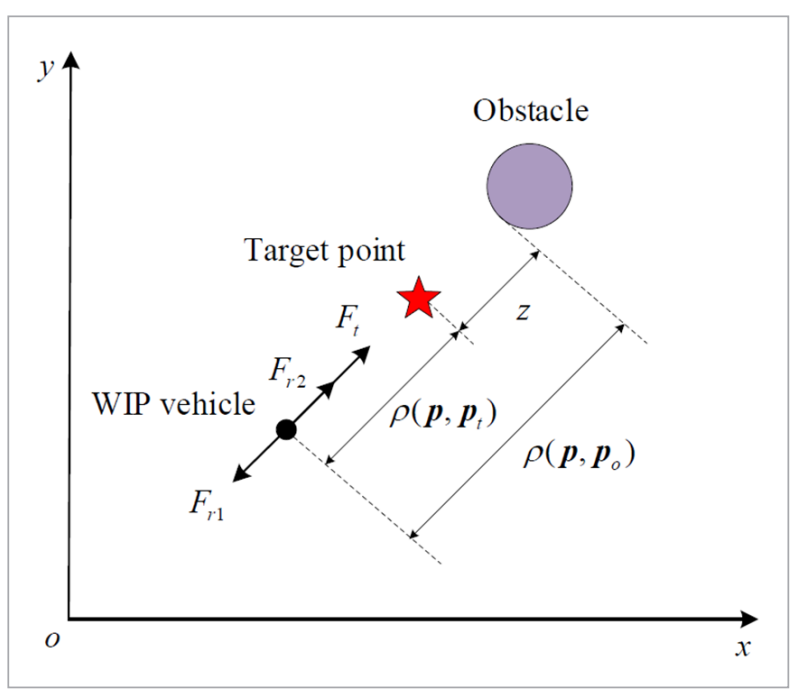

On that basis, rewrite Equations (8) - (9) as

$$
\begin{aligned}
& \boldsymbol{F}_{t}(\boldsymbol{p})=F_{t} \boldsymbol{n}_{1}, \\
& \boldsymbol{F}_{o}(\boldsymbol{p})=F_{r 1} \boldsymbol{n}_{2}+F_{r 2} \boldsymbol{n}_{1},
\end{aligned}
$$

where $\boldsymbol{n}_{1}=-\nabla \rho\left(\boldsymbol{p}, \boldsymbol{p}_{\mathrm{t}}\right), \boldsymbol{n}_{2}=\nabla \rho\left(\boldsymbol{p}, \boldsymbol{p}_{\mathrm{o}}\right), \boldsymbol{n}_{2}=-\boldsymbol{n}_{1}$, and $F_{\mathrm{t}}=k_{\mathrm{a}} \rho\left(\boldsymbol{p}, \boldsymbol{p}_{\mathrm{t}}\right)$.

Thus the total force acting on the WIP vehicle can be expressed as

$$
\boldsymbol{F}_{\text {total }}(\boldsymbol{p})=\left(F_{t}-F_{r 1}+F_{r 2}\right) \boldsymbol{n}_{1},
$$

To eliminate the free path local minima, $\boldsymbol{F}_{\text {total }}(\boldsymbol{p})$ should be pointing to the target, namely, $\boldsymbol{F}_{\text {total }}(\boldsymbol{p})$ has a same direction with $\boldsymbol{n}_{1}$, thus the following condition should be satisfied according to (14):

$$
F_{t}-F_{r 1}+F_{r 2}>0
$$

Substituting related Equations into (15) leads to

$$
\begin{aligned}
& k_{a} \rho\left(\boldsymbol{p}, \boldsymbol{p}_{t}\right)+\frac{k_{r} \varepsilon}{2}\left(\frac{1}{\rho\left(\boldsymbol{p}, \boldsymbol{p}_{o}\right)-\sigma}-\frac{1}{R_{o}-\sigma}\right)^{2} \rho^{\varepsilon-1}\left(\boldsymbol{p}, \boldsymbol{p}_{t}\right) \\
& -k_{r}\left(\frac{1}{\rho\left(\boldsymbol{p}, \boldsymbol{p}_{o}\right)-\sigma}-\frac{1}{R_{o}-\sigma}\right) \frac{\rho^{\varepsilon}\left(\boldsymbol{p}, \boldsymbol{p}_{t}\right)}{\left(\rho\left(\boldsymbol{p}, \boldsymbol{p}_{o}\right)-\sigma\right)^{2}}>0 .
\end{aligned}
$$

Since $k_{\mathrm{a}}, k_{\mathrm{r}}>0$, it can be written as

$$
\begin{aligned}
& \frac{k_{a}}{k_{r}}>\left(\frac{1}{\rho\left(\boldsymbol{p}, \boldsymbol{p}_{o}\right)-\sigma}-\frac{1}{R_{o}-\sigma}\right) \frac{\rho^{\varepsilon-1}\left(\boldsymbol{p}, \boldsymbol{p}_{t}\right)}{\left(\rho\left(\boldsymbol{p}, \boldsymbol{p}_{o}\right)-\sigma\right)^{2}}- \\
& \frac{\varepsilon}{2}\left(\frac{1}{\rho\left(\boldsymbol{p}, \boldsymbol{p}_{o}\right)-\sigma}-\frac{1}{R_{o}-\sigma}\right)^{2} \rho^{\varepsilon-2}\left(\boldsymbol{p}, \boldsymbol{p}_{t}\right) .
\end{aligned}
$$

Then, considering $\rho\left(\boldsymbol{p}, \boldsymbol{p}_{\mathrm{t}}\right)=\rho\left(\boldsymbol{p}, \boldsymbol{p}_{\mathrm{o}}\right)-z$, it can be further derived as

$$
\begin{aligned}
& \frac{k_{a}}{k_{r}}>\left(\frac{1}{\rho\left(\boldsymbol{p}, \boldsymbol{p}_{o}\right)-\sigma}-\frac{1}{R_{o}-\sigma}\right) \frac{\left(\rho\left(\boldsymbol{p}, \boldsymbol{p}_{t}\right)-z\right)^{\varepsilon-1}}{\left(\rho\left(\boldsymbol{p}, \boldsymbol{p}_{o}\right)-\sigma\right)^{2}}- \\
& \frac{\varepsilon}{2}\left(\frac{1}{\rho\left(\boldsymbol{p}, \boldsymbol{p}_{o}\right)-\sigma}-\frac{1}{R_{o}-\sigma}\right)^{2}\left(\rho\left(\boldsymbol{p}, \boldsymbol{p}_{o}\right)-z\right)^{\varepsilon-2} .
\end{aligned}
$$

Letting $h$ denote the supremum of the right-hand side of above formula and abbreviate $\rho\left(\boldsymbol{p}, \boldsymbol{p}_{\mathrm{o}}\right)$ as $\rho$, we have

$$
\begin{aligned}
h= & \sup _{z<\rho<R_{o}}\left\{\left(\frac{1}{\rho\left(\boldsymbol{p}, \boldsymbol{p}_{o}\right)-\sigma}-\frac{1}{R_{o}-\sigma}\right) \frac{\left(\rho\left(\boldsymbol{p}, \boldsymbol{p}_{o}\right)-z\right)^{\varepsilon-1}}{\left(\rho\left(\boldsymbol{p}, \boldsymbol{p}_{o}\right)-\sigma\right)^{2}}-\right. \\
& \left.\frac{\varepsilon}{2}\left(\frac{1}{\rho\left(\boldsymbol{p}, \boldsymbol{p}_{o}\right)-\sigma}-\frac{1}{R_{o}-\sigma}\right)^{2}\left(\rho\left(\boldsymbol{p}, \boldsymbol{p}_{o}\right)-z\right)^{\varepsilon-2}\right\} \\
= & \sup _{z<\rho<R_{o}}\left\{( \frac { 1 } { \rho - \sigma } - \frac { 1 } { R _ { o } - \sigma } ) ( \rho - z ) ^ { \varepsilon - 2 } \left[\frac{\rho}{(\rho-\sigma)^{2}}-\right.\right. \\
& \left.\left.\frac{z}{(\rho-\sigma)^{2}}-\frac{\varepsilon}{2(\rho-\sigma)}+\frac{\varepsilon}{2\left(R_{o}-\sigma\right)}\right]\right\}
\end{aligned}
$$

Since $z<\rho<R_{0}$, we have $\left[1 /(\rho-\sigma)-1 /\left(R_{0}-\sigma\right)\right]>0$ and $(\rho-\mathrm{z})^{\varepsilon-2}>0$. Next, we will detailedly analyze the sign of $\left\{\rho /(\rho-\sigma)^{2}-z /(\rho-\sigma)^{2}-\varepsilon /[2(\rho-\sigma)]+\varepsilon /\left[2\left(R_{0}-\sigma\right)\right]\right\}$. As a convenience, we first define

$$
\alpha(\rho)=: \frac{\rho}{(\rho-\sigma)^{2}}-\frac{z}{(\rho-\sigma)^{2}}-\frac{\varepsilon}{2(\rho-\sigma)}+\frac{\varepsilon}{2\left(R_{o}-\sigma\right)} .
$$

Then, through some algebraic operations, we can draw the following conclusion.

For $\varepsilon=2$, it holds that 


$$
\alpha(\rho)\left\{\begin{array}{l}
>0, \rho>\rho_{1} \\
\leq 0, \rho \leq \rho_{1}
\end{array},\right.
$$

where $\rho_{1}=\sigma+\sqrt{(z-\sigma)\left(R_{o}-\sigma\right)}$ that satisfies $z<\rho_{1}<R_{\mathrm{o}}$. In this situation, we obtain

$$
\begin{aligned}
h & \leq \sup _{\rho_{1}<\rho<R_{o}}\left[\left(\frac{1}{\rho-\sigma}-\frac{1}{R_{o}-\sigma}\right) \alpha(\rho)\right] \\
& \leq\left(\frac{1}{\rho_{1}-\sigma}-\frac{1}{R_{o}-\sigma}\right) \alpha\left(R_{o}\right) \\
& =\left(\frac{1}{\rho_{1}-\sigma}-\frac{1}{R_{o}-\sigma}\right) \frac{R_{o}-z}{\left(R_{o}-\sigma\right)^{2}}
\end{aligned}
$$

and denote

$$
h_{1}=\left(\frac{1}{\rho_{1}-\sigma}-\frac{1}{R_{o}-\sigma}\right) \frac{R_{o}-z}{\left(R_{o}-\sigma\right)^{2}} .
$$

For $\varepsilon>2$, there is

$$
\alpha(\rho)\left\{\begin{array}{l}
>0, \rho>\rho_{2} \\
\leq 0, \rho \leq \rho_{2}
\end{array},\right.
$$

where

$\rho_{2}=\sigma+2(z-\sigma) /\left[1-\frac{\varepsilon}{2}+\sqrt{\left(1-\frac{\varepsilon}{2}\right)^{2}+\frac{2 \varepsilon(z-\sigma)}{R_{o}-\sigma}}\right]$.

Thus we can obtain

$$
\begin{aligned}
h & \leq \sup _{\rho_{2}<\rho<R_{o}}\left[\left(\frac{1}{\rho-\sigma}-\frac{1}{R_{o}-\sigma}\right)(\rho-z)^{\varepsilon-2} \alpha(\rho)\right] \\
& \leq\left(\frac{1}{\rho_{2}-\sigma}-\frac{1}{R_{o}-\sigma}\right)\left(R_{o}-z\right)^{\varepsilon-2} \alpha\left(R_{o}\right), \\
& =\left(\frac{1}{\rho_{2}-\sigma}-\frac{1}{R_{o}-\sigma}\right) \frac{\left(R_{o}-z\right)^{\varepsilon-1}}{\left(R_{o}-\sigma\right)^{2}}
\end{aligned}
$$

and denote

$$
h_{2}=\left(\frac{1}{\rho_{2}-\sigma}-\frac{1}{R_{o}-\sigma}\right) \frac{\left(R_{o}-z\right)^{\varepsilon-1}}{\left(R_{o}-\sigma\right)^{2}} .
$$

For $1 \leq \varepsilon<2$, it follows that

$$
\alpha(\rho)\left\{\begin{array}{l}
>0, \quad \rho_{3}<\rho<\rho_{4}, \\
\leq 0, \rho \leq \rho_{3} \text { or } \rho \geq \rho_{4}
\end{array},\right.
$$

and

$$
\sup _{\rho_{3}<\rho<\rho_{4}} \alpha(\rho)=\alpha\left(\rho_{5}\right)
$$

where

$$
\begin{aligned}
& \rho_{3}=\sigma+2(z-\sigma) /\left[1-\frac{\varepsilon}{2}+\sqrt{\left(1-\frac{\varepsilon}{2}\right)^{2}+\frac{2 \varepsilon(z-\sigma)}{R_{o}-\sigma}}\right] \\
& \rho_{4}=\sigma+2(z-\sigma) /\left[1-\frac{\varepsilon}{2}-\sqrt{\left(1-\frac{\varepsilon}{2}\right)^{2}+\frac{2 \varepsilon(z-\sigma)}{R_{o}-\sigma}}\right]
\end{aligned}
$$

and $\rho_{5}=(1-\varepsilon / 2) /[2(z-\sigma)]$. Hence we can obtain

$$
\begin{aligned}
h & \leq \sup _{\rho_{3}<\rho<\rho_{4}}\left[\left(\frac{1}{\rho_{3}-\sigma}-\frac{1}{R_{o}-\sigma}\right)\left(\rho_{3}-z\right)^{\varepsilon-2} \alpha(\rho)\right] \\
& \leq\left(\frac{1}{\rho_{3}-\sigma}-\frac{1}{R_{o}-\sigma}\right)\left(\rho_{3}-z\right)^{\varepsilon-2} \sup _{\rho_{3}<\rho<\rho_{4}} \alpha(\rho), \\
& =\left(\frac{1}{\rho_{3}-\sigma}-\frac{1}{R_{o}-\sigma}\right)\left(\rho_{3}-z\right)^{\varepsilon-2} \alpha\left(\rho_{5}\right)
\end{aligned}
$$

and denote

$$
h_{3}=\left(\frac{1}{\rho_{3}-\sigma}-\frac{1}{R_{o}-\sigma}\right)\left(\rho_{3}-z\right)^{\varepsilon-2} \alpha\left(\rho_{5}\right) .
$$

Therefore, as long as we choose $k_{\mathrm{a}} / k_{\mathrm{r}}>h_{1}$ when $\varepsilon=2, k_{\mathrm{a}} /$ $k_{\mathrm{r}}>h_{2}$ when $\varepsilon>2$, and $k_{\mathrm{a}} / k_{\mathrm{r}}>h_{3}$ when $1 \leq \varepsilon<2$, there will be no local minima occur. To prove this point, returning to our example shown in Figure 1, it can be calculated that $h_{2}=0.1675$ from (26), which cannot satisfy $k_{\mathrm{a}} / k_{\mathrm{r}}>h_{2}$ with $k_{\mathrm{a}}=2$ and $k_{\mathrm{r}}=100$, hence there exist local

\section{Figure 6}

Total potential without local minimum

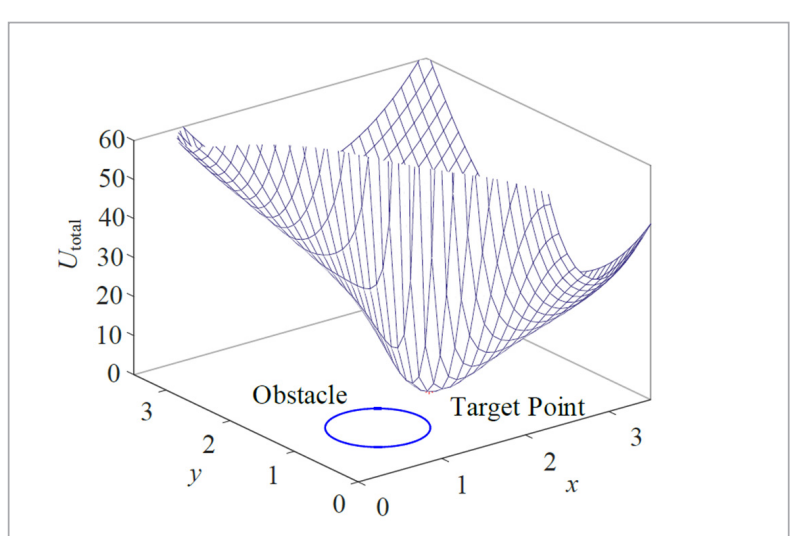


minima. Furthermore, we choose $k_{\mathrm{a}}=20$ and $k_{\mathrm{r}}=100$ that satisfy $k_{\mathrm{a}} / k_{\mathrm{r}}>h_{2}$, and the other parameters remain unchanged, then the total potential is shown in Figure 6 . In addition, the total potential on the line passing through the target point and the nearest boundary point on the obstacle is shown in Figure 7, from which we can see that the local minimum in Figure 3 can be eliminated successfully.

\section{Figure 7}

Total potential on the line without local minimum

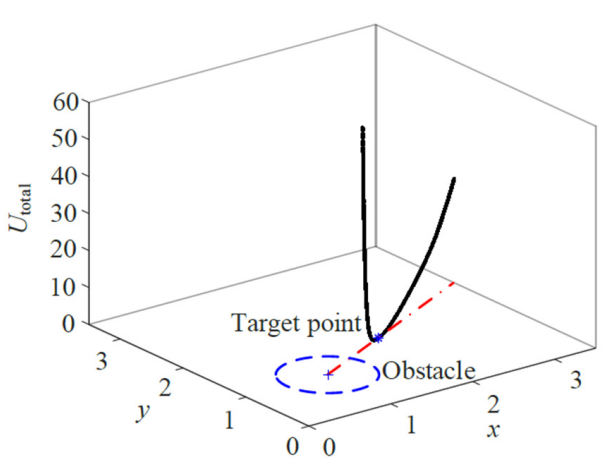

\section{Controller Design Based on IAPF}

In the motion environment, there may be more obstacles in the detection range $\rho_{0}$ of the WIP vehicle, and the resultant force imposing on the vehicle can be expressed as

$$
\boldsymbol{F}_{r}(\boldsymbol{p})=\boldsymbol{F}_{t}(\boldsymbol{p})+\sum_{i=1}^{N} \boldsymbol{F}_{o i}(\boldsymbol{p}),
$$

where $F_{\mathrm{oi}}(\boldsymbol{p})$ represents the acting force of the $i$ th obstacle on the vehicle; $N=1,2,3, \ldots$ is the number of obstacles around the vehicle.

The WIP vehicle subject to nonholonomic constraint (1) cannot generate acceleration in arbitrary direction, and only can be controlled by the steering motion and longitudinal motion, as shown in Figure 8. Therefore, the direction information of the resultant force $\boldsymbol{F}_{\mathrm{r}}(\boldsymbol{p})$ is used to control the steering motion of the vehicle, and the magnitude information of $\boldsymbol{F}_{\mathrm{r}}(\boldsymbol{p})$ is used to control the longitudinal motion of the vehicle in this paper. The designed controller for the obstacles avoidance control of the WIP vehicle is as follows: $\tau_{\omega}=k_{1}(\delta-\varphi)+k_{2}(\dot{\delta}-\dot{\varphi})$,

$\tau_{v}=k_{3}\left(x_{v}-k I_{r}\right)+k_{4}\left(\dot{x}_{v}-k F_{r}\right)+k_{5} \theta+k_{6} \dot{\theta}$,

where $I_{r}=\int F_{r} \mathrm{~d} t, \delta$ represents the angle from the direction of $x_{\mathrm{v}}$ to $F_{\mathrm{r}} ; k_{1}, k_{2}, k_{3}, k_{4}, k_{5}$ and $k_{6}$ are seven positive parameters; $k$ is a non-negative design parameter.

\section{Figure 8}

Motion decomposition of the WIP vehicle

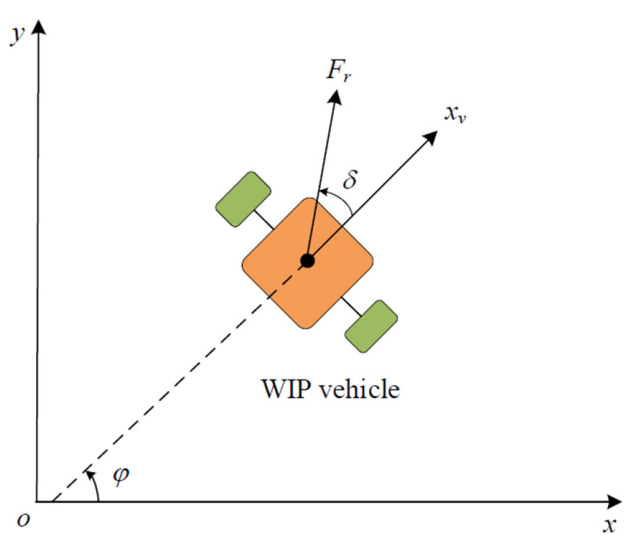

Next, we will clarify that the proposed controller can ensure the stability of the underactuated vehicle body while conducting an obstacles avoidance motion simultaneously. According to the driving equation of the WIP vehicle, we can obtain the following formula with the rolling resistance and air resistance being neglected:

$$
\tau_{v}=\kappa\left(2 m_{w}+m_{c}+m_{b}\right) r \ddot{x}_{v},
$$

where $\kappa$ denotes the conversion coefficient of rotating mass, which satisfies $k>1$ and takes $\kappa=1.1$ in this paper. Combining (33) and (34), we obtain

$$
\ddot{x}_{v}=\lambda_{1} u+\lambda_{2} \theta+\lambda_{3} \dot{\theta},
$$

where $\lambda_{1}=k_{3} / \Upsilon, u=x_{v}-k I_{r}+k_{4}\left(\dot{x}_{v}-k F_{r}\right) / k_{3}, \lambda_{2}=k_{5} / \Upsilon$, $\lambda_{3}=k_{6} / Y, Y=\kappa\left(2 m_{v}+m_{c}+m_{b}\right) r$.

Substituting (35) into (4), we obtain

$$
\frac{2 J_{2}}{m_{b} L} \ddot{\theta}+\lambda_{3} \dot{\theta}+\left(\lambda_{2}-g\right) \theta=-\lambda_{1} u \text {. }
$$


Let $a_{1}=2 J_{2} /\left(m_{b} L \lambda_{3}\right), a_{2}=\left(\lambda_{3}-g\right) / \lambda_{3}, a_{3}=-\lambda_{1} / \lambda_{3}$, and then the (36) can be expressed as

$$
a_{1} \ddot{\theta}+\dot{\theta}+a_{2} \theta=a_{3} u \text {. }
$$

Regarding $u$ and $\theta$ as the input and output, respectively, the transfer function of (37) can be formulated as

$$
\phi(s)=\frac{a_{3}}{a_{1} s^{2}+s+a_{2}} .
$$

Let the denominator polynomial of (38) is zero, and then the characteristic equation of system (37) can be obtained, as follows:

$$
a_{1} s^{2}+s+a_{2}=0 .
$$

Hence, the two characteristic roots can be calculated out, as follows:

$$
s_{1,2}=-\frac{1}{2 a_{1}} \pm \frac{\sqrt{1-4 a_{1} a_{2}}}{2 a_{1}} .
$$

According to the related control theory, the necessary and sufficient condition for the system stability is that all the characteristic roots have negative real parts [19], which means the following relationship should be satisfied:

$$
1-4 a_{1} a_{2} \leq 0 \text { and } a_{1}>0,
$$

or

$$
1-4 a_{1} a_{2}>0 \text { and }-\frac{1}{2 a_{1}} \pm \frac{\sqrt{1-4 a_{1} a_{2}}}{2 a_{1}}<0 .
$$

Therefore, the proposed controller can ensure the stability of the vehicle body, provided that $a_{1}$ and $a_{2}$ are selected to satisfy (41) or (42).

\section{Parameter Adjustment with Fuzzy Logic}

In this section, some simulation and mathematical analyses are firstly performed to illustrate the necessity of the parameter adjustment, and then a fuzzy regulator is presented to adjust the key control parameter to balance the stability and efficiency of the proposed controller.

\subsection{Analysis of Necessity}

To preliminarily validate the feasibility of the controller proposed in previous section, a simulation study is performed in Matlab/Simulink platform. Simulation parameters that satisfy the related requirements given in the previous sections, such as (41) or (42), etc., are selected as follows: $\boldsymbol{q}_{0}=[1.2,0.2,0,0]^{\mathrm{T}}, \boldsymbol{q}_{\mathrm{f}}=[1.7,3.7$, $-, 0]^{\mathrm{T}}, k_{\mathrm{a}}=1, k_{\mathrm{r}}=0.1, \rho_{0}=1, \sigma=0.01, k=3, k_{1}=1, k_{2}=0.1, k_{3}=0.1$, $k_{4}=0.3, k_{5}=10, k_{6}=2$, where "-" in $\boldsymbol{q}_{\mathrm{f}}$ represents that $\varphi_{\mathrm{f}}$ can be arbitrary value. In addition, the size and distribution of obstacles are shown in Figure 9, and the limit distances of the repulsive potential field influence are chosen as three times of the obstacle radii, respectively.

\section{Figure 9}

Motion path in multi-obstacle environment

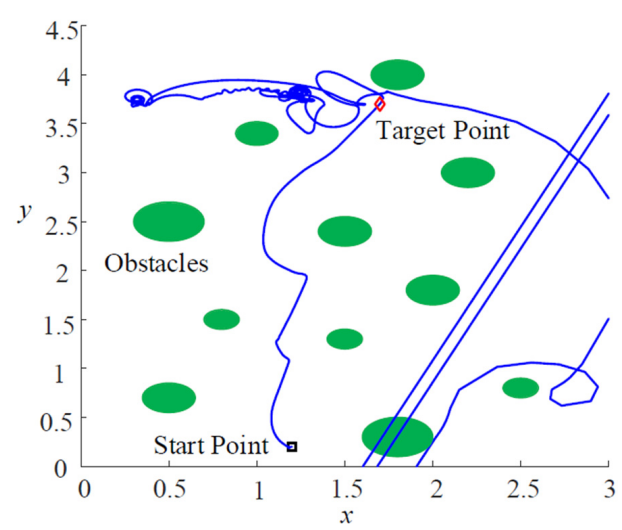

The motion path of the vehicle is shown in Figure 9, where it suggests that the vehicle can avoid several obstacles initially, but cannot remain stable at the target point and eventually move to a distance. Moreover, it can be seen from Figure 10 that the state variables $x, y$ and $\theta$ all diverge to a big value at about $3.8 \mathrm{~s}$, which means a failure to control the WIP vehicle in multi-obstacle environment. This is mainly because the designed controller is formulated based on the assumption that $\theta$ remains in a small region, but the stability condition (41) or (42) can only ensure $\theta$ converge to a certain value rather than a small value. 


\section{Figure 10}

Time response of $x, \mathrm{y}$ and $\theta$

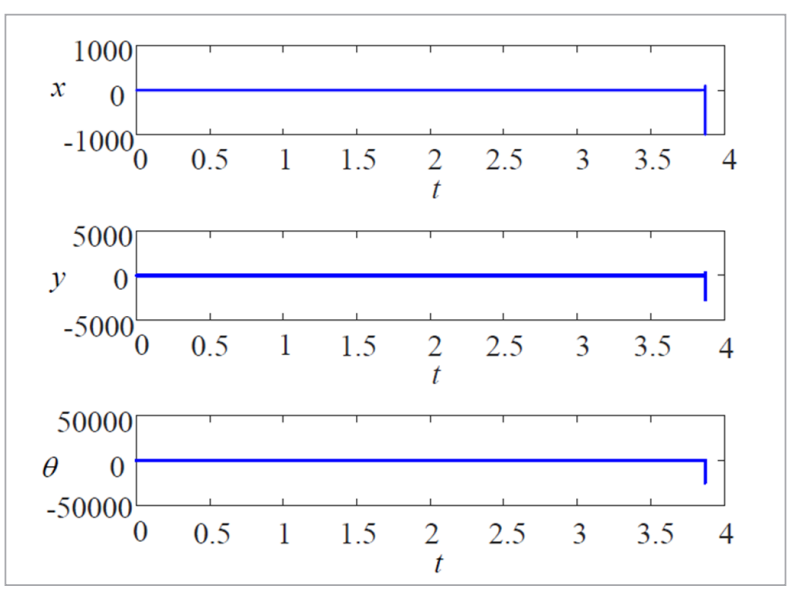

To facilitate the deduce, let $\omega_{n}=\sqrt{a_{2} / a_{1}}$ and $\varsigma=1 /\left(2 \sqrt{a_{1} a_{2}}\right)$, and then (38) can be rewritten as

$$
\phi(s)=\frac{a_{3}}{a_{2}} \frac{\omega_{n}^{2}}{s^{2}+2 \varsigma \omega_{n} s+\omega_{n}^{2}} .
$$

According to the related control theory [19], the system is stable if $\zeta>0$, and if the system response to a step input fulfills the requirements, the responses to other inputs also can satisfy the requirements. Therefore, consider a step input $U(s)=\beta(s)$ and suppose $0<\zeta<1$, and then the Laplace transformation of the system output can be expressed as

$$
\Theta(s)=\frac{a_{3}}{a_{2}} \frac{\omega_{n}^{2}}{s^{2}+2 \varsigma \omega_{n} s+\omega_{n}^{2}} \frac{\beta}{s} .
$$

To calculate the system response, the further deduction is conducted, as follows

$$
\begin{aligned}
\Theta(s)= & \frac{\beta a_{3}}{a_{2}}\left[\frac{1}{s}-\frac{s+2 \zeta \omega_{n}}{\left(s+\zeta \omega_{n}\right)^{2}+\left(1-\zeta^{2}\right) \omega_{n}^{2}}\right] \\
= & \frac{\beta a_{3}}{a_{2}}\left[\frac{1}{s}-\frac{s+\zeta \omega_{n}}{\left(s+\zeta \omega_{n}\right)^{2}+\left(1-\zeta^{2}\right) \omega_{n}^{2}}-\right. \\
& \left.\frac{\zeta}{\sqrt{1-\zeta^{2}}} \frac{\sqrt{1-\zeta^{2}} \omega_{n}}{\left(s+\zeta \omega_{n}\right)^{2}+\left(1-\zeta^{2}\right) \omega_{n}^{2}}\right]
\end{aligned}
$$

Performing an inverse Laplace transformation for (45), we obtain

$$
\begin{aligned}
\theta(t)= & \frac{\beta a_{3}}{a_{2}}\left[1-e^{-\zeta \omega_{n} t} \cos \left(\sqrt{1-\zeta^{2}} \omega_{n} t\right)-\right. \\
& \left.\frac{\zeta}{\sqrt{1-\zeta^{2}}} e^{-\zeta \omega_{n} t} \sin \left(\sqrt{1-\zeta^{2}} \omega_{n} t\right)\right] \\
= & \frac{\beta a_{3}}{a_{2}}-\frac{\beta a_{3} e^{-\zeta \omega_{n} t}}{a_{2} \sqrt{1-\zeta^{2}}} \sin \left(\sqrt{1-\zeta^{2}} \omega_{n} t+\right. \\
& \left.\arctan \frac{\sqrt{1-\zeta^{2}}}{\zeta}\right)
\end{aligned} .
$$

From (46), it can be seen that the system response to the unit step input contains two parts: one is the steady-state value $\beta \mathrm{a}_{3} / a_{2}$; the other is a damped sinusoid component that decays with time. Thus $\left|\beta \mathrm{a}_{3}\right|$ $a_{2} \mid$ should be designed small enough to ensure $|\theta|$ remains in a small region, and there is a similar conclusion if $\zeta=1$ or $\zeta>1$. According to the derivation in previous section, the parameter $k$ plays an important role in the value $|\beta|$, and a smaller $k$ corresponds to a smaller $|\beta|$. With this in mind, we set $k$ to a smaller value 0.1 and keep the other parameters unchanged, and then the simulation results are shown in Figures 1112. From Figure 11, it can be seen that the WIP vehicle can arrive the target point from the start point successfully, and no any collision occurs. In addition, we can see from Figure 12 that the position of the vehicle $(x, y)$ can reach the target position $(1.7,3.7)$ at about

\section{Figure 11}

Motion path in multi-obstacle environment with smaller $k$

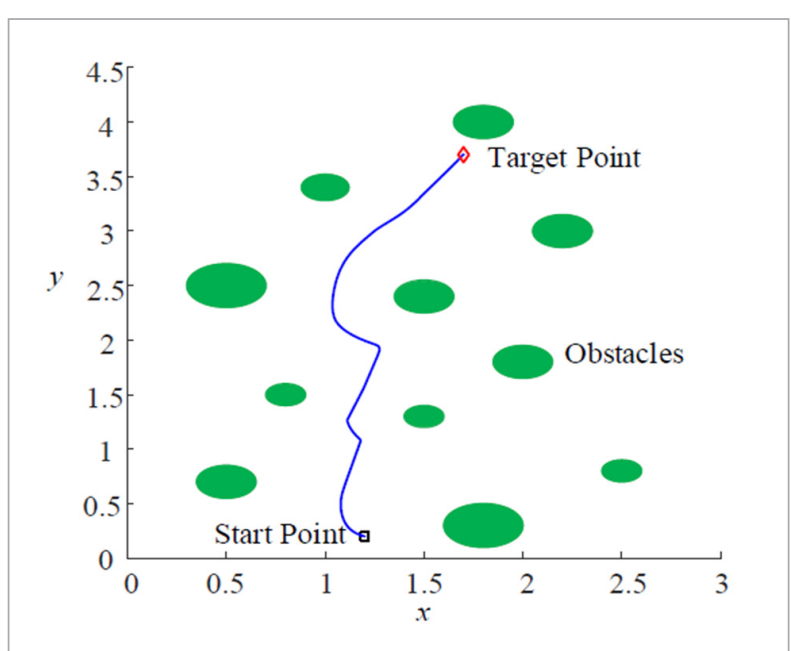


Figure 12

Time response of $x, y$, and $\theta$ with smaller $k$
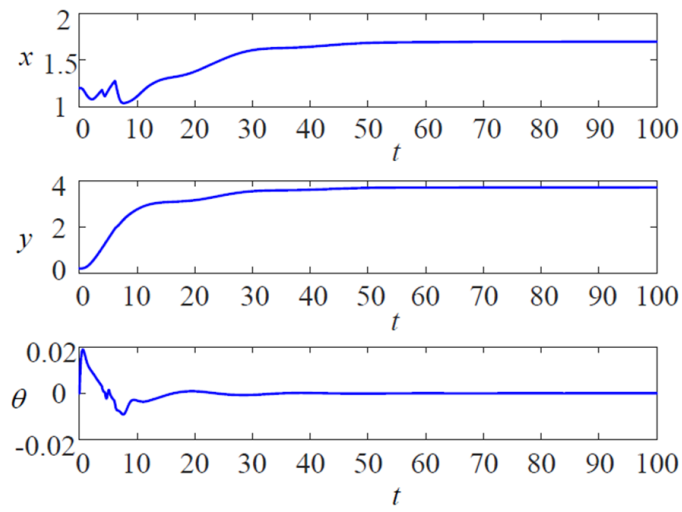

50s and remain at this point stably, and the tilt angle of vehicle body $\theta$ keeps a small value all the time. On the basis of these studies, it can be concluded that the smaller $k$ is, the better control stability and the lower control efficient are, thus it is necessary to adjust the key parameter $k$ in the motion process according to the motion environment timely to strike a better balance between the control efficient and stability.

\subsection{Design of the Fuzzy Regulator}

The influence of motion environment on the WIP vehicle is mainly embodied in the resultant force $F_{r}$, and generally the bigger $F_{\mathrm{r}}$ is, the smaller $k$ should be. Moreover, the stability of the WIP vehicle is mainly reflect in the absolute value of the tilt angle $|\theta|$, and usually the bigger $|\theta|$ is, the smaller $k$ should be. However, it is difficult to formulate these relationships as exact mathematical formulas, thus the fuzzy logic is adopted to design the parameter regulator in this study due to the outstanding merit of describing the linguistic knowledge [1, 31, 39].

To this end, define three fuzzy sets "Big- $F_{\mathrm{r}}$, "Medium$F_{\mathrm{r}}$ " and "Small- $F_{\mathrm{r}}$ " for $F_{\mathrm{r}}$ from experience, and the membership functions of which can be expressed with $\mu_{\mathrm{B}}\left(F_{\mathrm{r}}\right), \mu_{\mathrm{M}}\left(F_{\mathrm{r}}\right), \mu_{\mathrm{S}}\left(F_{\mathrm{r}}\right)$, respectively; define three fuzzy sets "Big- $|\theta|$ ", "Medium- $|\theta|$ " and "Small- $|\theta|$ " for $|\theta|$, and the membership functions of which can be expressed with $\mu_{\mathrm{B}}(|\theta|), \quad \mu_{\mathrm{M}}(|\theta|), \quad \mu_{\mathrm{S}}(|\theta|)$, respectively; define three fuzzy sets "Big- $k$ ", "Medium- $k$ " and "Small- $k$ " for $k$, and the membership functions of which can be expressed with $\mu_{\mathrm{B}}(k), \mu_{\mathrm{M}}(k), \mu_{\mathrm{S}}(k)$, respectively.
Referring to the literature [31] and according to some empirical knowledge, the expressions of these membership functions are chosen as follows:

$$
\begin{aligned}
& \mu_{B}\left(F_{r}\right)=\frac{1}{1+e^{-5\left(F_{r}-6\right)}}, \mu_{M}\left(F_{r}\right)=e^{-0.2\left(F_{r}-4\right)^{2}}, \\
& \mu_{S}\left(F_{r}\right)=\frac{1}{1+e^{5\left(F_{r}-2\right)}}, \mu_{B}(|\theta|)=\frac{1}{1+e^{-100(|\theta|-0.1)},} \\
& \mu_{M}(|\theta|)=e^{-1000(|\theta|-0.075)^{2}}, \mu_{S}(|\theta|)=\frac{1}{1+e^{100(|\theta|-0.05)}}, \\
& \mu_{B}(k)=e^{-200(k-0.12)^{2}}, \mu_{M}(k)=e^{-200(k-0.06)^{2}}, \\
& \mu_{S}(k)=e^{-200 k^{2}} .
\end{aligned}
$$

Then, nine fuzzy rules are designed based on the linguistic knowledge mentioned above, as shown in Table 1.

\section{Table 1}

Fuzzy inference rules

\begin{tabular}{c|c|c|c}
\hline Number & $F_{\mathrm{r}}$ & $|\theta|$ & $k$ \\
\hline 1 & Big- $F_{\mathrm{r}}$ & Big- $|\theta|$ & Small- $k$ \\
\hline 2 & Big- $F_{\mathrm{r}}$ & Medium- $|\theta|$ & Small- $k$ \\
\hline 3 & Big- $F_{\mathrm{r}}$ & Small- $|\theta|$ & Medium- $k$ \\
\hline 4 & Medium- $F_{\mathrm{r}}$ & Big- $|\theta|$ & Small- $k$ \\
\hline 5 & Medium- $F_{\mathrm{r}}$ & Medium- $|\theta|$ & Medium- $k$ \\
\hline 6 & Medium- $F_{\mathrm{r}}$ & Small- $|\theta|$ & Medium- $k$ \\
\hline 7 & Small- $F_{\mathrm{r}}$ & Big- $|\theta|$ & Small- $k$ \\
\hline 9 & Small- $F_{\mathrm{r}}$ & Medium- $|\theta|$ & Medium- $k$ \\
\hline & Small- $F_{\mathrm{r}}$ & Small- $|\theta|$ & Big- $k$ \\
\hline
\end{tabular}

On that basis, the fuzzy regulator for the key parameter $k$ can be derived with product inference and center-average defuzzifier, as follows.

$$
k=\frac{\varpi_{1}+\varpi_{2}}{\Delta},
$$

where

$\varpi_{1}=0.06\left[\mu_{B}\left(F_{r}\right) \mu_{S}(|\theta|)+\mu_{M}\left(F_{r}\right) \mu_{M}(|\theta|)+\right.$, $\left.\mu_{M}\left(F_{r}\right) \mu_{S}(|\theta|)+\mu_{S}\left(F_{r}\right) \mu_{M}(|\theta|)\right]$

$\varpi_{2}=0.12 \mu_{S}\left(F_{r}\right) \mu_{S}(|\theta|)$, 


$$
\begin{aligned}
\Delta= & \mu_{B}\left(F_{r}\right) \mu_{B}(|\theta|)+\mu_{B}\left(F_{r}\right) \mu_{M}(|\theta|)+ \\
& \mu_{M}\left(F_{r}\right) \mu_{B}(|\theta|)+\mu_{S}\left(F_{r}\right) \mu_{B}(|\theta|)+ \\
& \mu_{B}\left(F_{r}\right) \mu_{S}(|\theta|)+\mu_{M}\left(F_{r}\right) \mu_{M}(|\theta|)+ \\
& \mu_{M}\left(F_{r}\right) \mu_{S}(|\theta|)+\mu_{S}\left(F_{r}\right) \mu_{M}(|\theta|)+ \\
& \mu_{S}\left(F_{r}\right) \mu_{S}(|\theta|)
\end{aligned}
$$

Up to now, the control method for a WIP vehicle in multi-obstacle environment has been presented completely, which will be further validated in the next section.

\section{Simulation Analysis}

To demonstrate the control performance of the proposed method, some numerical simulations are carried out in Matlab/Simulink environment on a computing platform that contains an Intel Pentium G4560 processor running at $3.5 \mathrm{~Hz}$ and $8 \mathrm{~GB}$ of RAM. The motion environment are set as a more complex situation than that in previous section, where multiple types of obstacles, such as dense obstacles, narrow corridor and an obstacle near target, are all considered. Specifically, to simulate the obstacle detection in real time, the position coordinates of all obstacles are prestored in the control program, and the obstacle will be deemed to be in the detection range of the vehicle if the distance between the obstacle and the vehicle is less than or equal $\rho_{0}$. In addition, to illustrate the importance of the parameter adjustment with fuzzy logic, the simulations are conducted in three cases, i.e., $k=0.08, k=0.8$, and $k$ adjusted by fuzzy logic; the other parameters remain consistent with that in previous section, and the results are shown in Figures 13-14.

It can be seen that there occur many collisions if $k$ is set as a larger value 0.8 , whereas the vehicle can reach the target point stably if $k$ is given as a smaller value 0.08 or adjusted based on the fuzzy regulator, as shown in Figure 13 with blue dash-dot line, black solid line and fuchsia dashed line, respectively. Furthermore, it tells that $x, y$ and $\theta$ in the case of $k=0.8$ diverge to a big unexpected values, which means the obstacles avoidance task is not accomplished, as shown in Figure 14 with black dash-dot line. On the contrary, $x$ and $y$ can converge to the target values if $k=0.08$ or $k$ is adjusted by fuzzy logic at about $77 \mathrm{r}$ and $54 \mathrm{~s}$, as shown in Figure 14 with solid red line and blue dashed line, respective-
Figure 13

Motion path in three cases

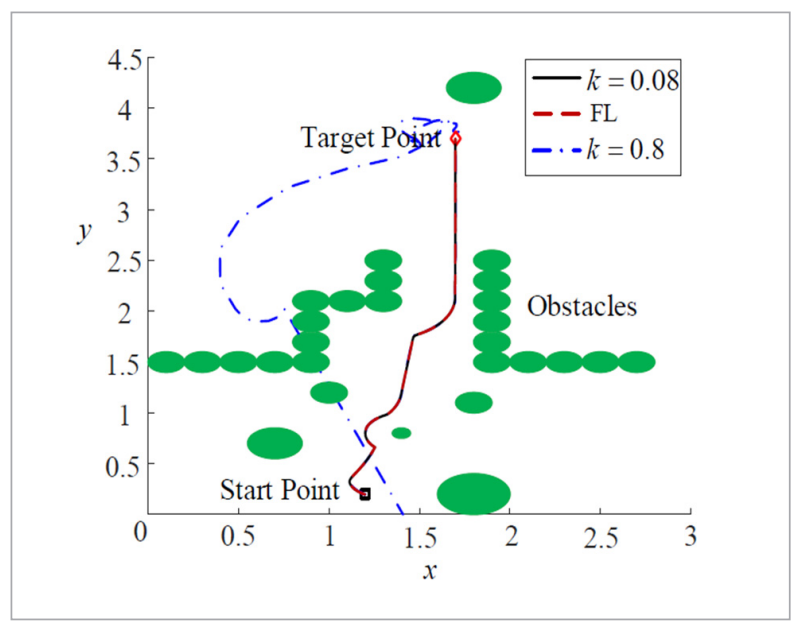

Figure 14

Time response of $x, y$ and $\theta$ in three cases

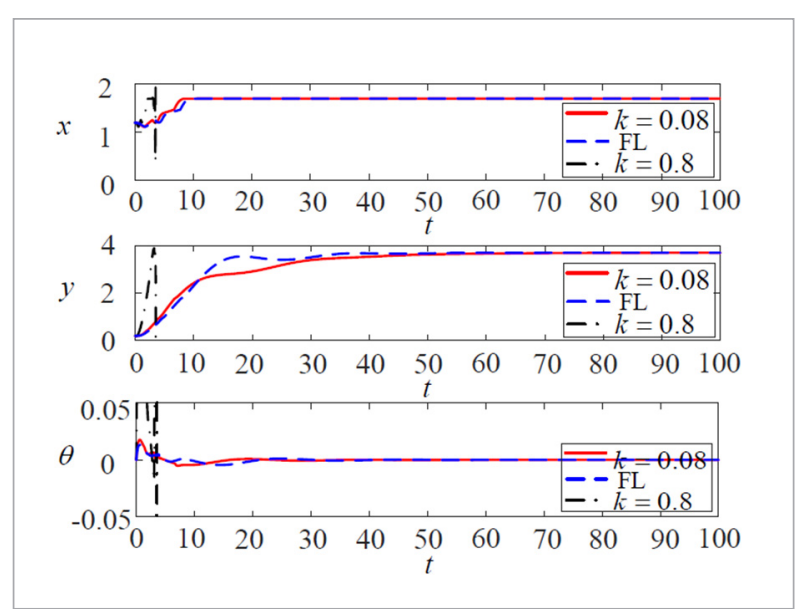

ly, which means the controller with fuzzy regulator is more efficient. It also can be seen that the absolute value of $\theta$ remains in a small region (less than 0.05) in both cases, which means the vehicle can move stably. Specifically, the time response of $\mathrm{k}$ regulated by fuzzy logic is presented in Figure 15, and the corresponding control inputs are shown in Figure 16. In addition, the stability and convergence time in three cases are compared in Table 2 for clarity, which indicates that the presented method can strike a good balance between control efficient and stability, and perform the obstacles avoidance task successfully. 
Figure 15

Time response of $k$ regulated by fuzzy logic

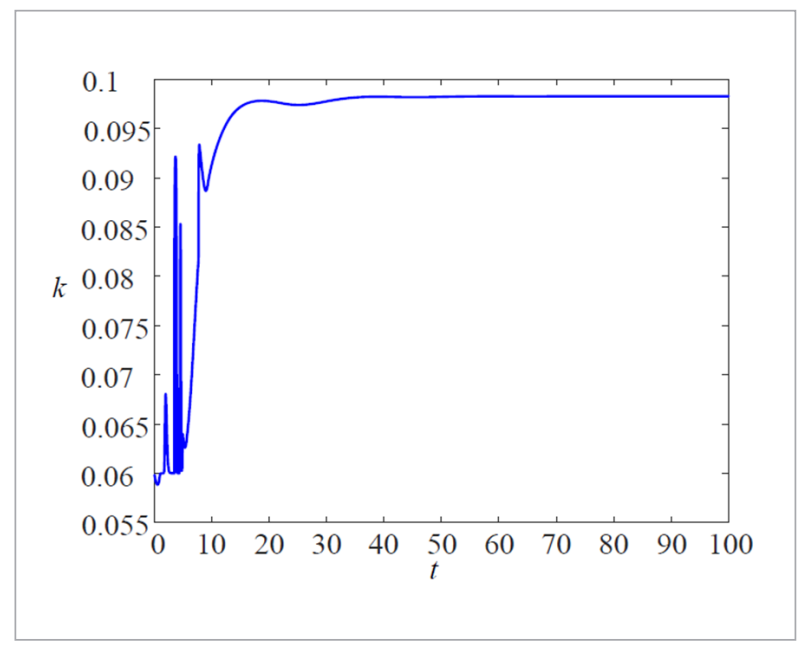

Figure 16

Control inputs

Control inputs
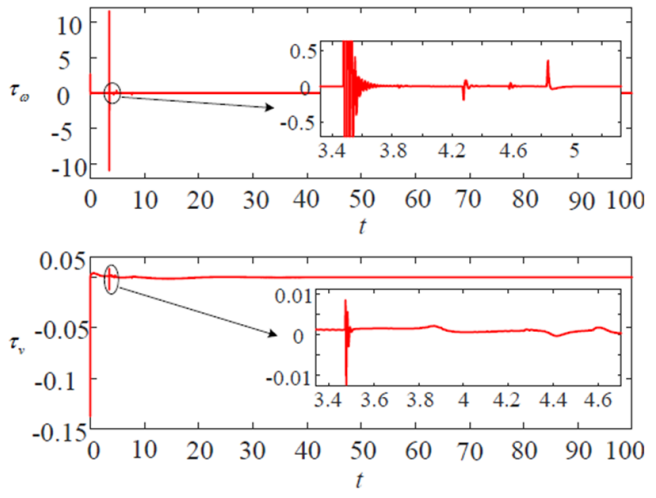

Table 2

Comparison of stability and convergence time

\begin{tabular}{c|c|c}
\hline Case & Stability & Convergence Time \\
\hline$k=0.08$ & Stable & $77 \mathrm{~s}$ \\
\hline FL & Stable & $54 \mathrm{~s}$ \\
\hline$k=0.8$ & Unstable & $\infty$ \\
\hline
\end{tabular}

\section{Conclusion}

In this work, a control method based on IAPF is proposed for the WIP vehicle in multi-obstacle environment. Specifically, a safe distance is introduced to the existing repulsive potential field to develop IAPF, by which the security issue and the probable local minima caused by the obstacle around the target can be solved simultaneously. On that basis, the obstacle avoidance controller for the WIP vehicle is designed based on IAPF, where the nonholonomic and underactuated characteristics are fully considered. Furthermore, an adjustment approach based on fuzzy logic is presented for the key parameter of the controller, hence the stability and efficiency of the control method are both concerned. Finally, adequate numerical simulations are conducted on a professional computing platform, and the results demonstrate the good performance of the proposed method.

\section{Acknowledgement}

This work was supported by National Natural Science Foundation of China (Nos. 61873047, 61874019, 51975089 and 91948203), Fundamental Research Funds for the Central Universities (DUT19ZD205), and State Key Laboratory of Robotics and System (SKLRS-2019-KF-17).

Information Technology and Control, 2019, 48(2), 179194. https://doi.org/10.5755/j01.itc.48.2.21390

3. Chan, R. P. M., Stol, K. A., Halkyard, C. R. Review of Modelling and Control of Two-Wheeled Robots. Annual Reviews in Control, 2013, 37(1), 89-103. https://doi. org/10.1016/j.arcontrol.2013.03.004

4. Chen, H. D., Chang, K. C., Agate, C. S. UAV Path Planning with Tangent-Plus-Lyapunov Vector Field Guidance od for Mobile Robots in Partially Known Environment. 
and Obstacle Avoidance. IEEE Transactions on Aerospace and Electronic Systems, 2013, 49(2), 840-856. https://doi.org/10.1109/TAES.2013.6494384

5. Cui, R.X., Guo, J., Mao, Z. Y. Adaptive Backstepping Control of Wheeled Inverted Pendulums Models. Nonlinear Dynamics, 2015, 79, 501-511. https://doi.org/10.1007/ s11071-014-1682-9

6. Ding, L., Li. S., Liu, Y. J., Gao, H. B., Chen, C., Deng, Z. Q. Adaptive Neural Network-Based Tracking Control for Full-State Constrained Wheeled Mobile Robotic System. IEEE Transactions on Systems, Man, and Cybernetics: Systems, 2017, 47(8), 2410-2419. https://doi. org/10.1109/TSMC.2017.2677472

7. Erkol, H. O. Optimal PI $\lambda \mathrm{D} \mu$ Controller Design for Two Wheeled Inverted Pendulum. IEEE Access, 2018, 6, 75709-75717. https://doi.org/10.1109/ACCESS.2018.2883504

8. Gajbhiye, S., Banavar, R. N., Delgado, S. Symmetries in the Wheeled Inverted Pendulum Mechanism. Nonlinear Dynamics, 2017, 90, 391-403. https://doi. org/10.1007/s11071-017-36r70-3

9. Ge, S. S., Cui, Y. J. New Potential Functions for Mobile Robot Path Planning. IEEE Transactions on Robotics and Automation, 2000, 16(5), 615-620. https://doi. org/10.1109/70.880813

10. Gehrig, S. K., Stein, F. J. Collision Avoidance for Vehicle-Following Systems. IEEE Transactions on Intelligent Transportation Systems, 2007, 8(2), 233-244. https://doi.org/10.1109/TITS.2006.888594

11. Ghasemi, H., Rezaie, B., Rahmani, Z. Terminal Sliding Mode Control with Evolutionary Algorithms for Finite-Time Robust Tracking of Nonholonomic Systems. Information Technology and Control, 2018, 47(1), 2644. https://doi.org/10.5755/j01.itc.47.1.15031

12. Huang, J., Ding, F., Fukuda, T., Matsuno, T. Modeling and Velocity Control for a Narrow Vehicle Based on Mobile Wheeled Inverted Pendulum. IEEE Transactions on Control Systems Technology, 2013, 21(5), 1607-1617. https://doi.org/10.1109/TCST.2012.2214439

13. Huang, J., Ri, S., Liu, L., Wang, Y. J., Kim, J., Pak, G. Nonlinear Disturbance Observer-Based Dynamic Surface Control of Mobile Wheeled Inverted Pendulum. IEEE Transactions on Control Systems Technology, 2015, 23(6), 2400-2407. https://doi.org/10.1109/ TCST.2015.2404897

14. Huang, J., Zhang, M. S., Ri, S., Xiong, C. H., Li, Z. J., Kang, Y. High-Order Disturbance Observer Based Sliding Mode Control for Mobile Wheeled Inverted Pendulum Systems. IEEE Transactions on Industrial Electron- ics, 2020, 67(3), 2030-2041. https://doi.org/10.1109/ TIE.2019.2903778

15. Jeong, S., Takahashi, T. Wheeled Inverted Pendulum Type Assistant Robot: Design Concept and Mobile Control. Intelligent Service Robotics, 2008, 1(4), 313-320. https://doi.org/10.1007/s11370-008-0024-5

16. Khatib, O. Real-Time Obstacle Avoidance for Manipulators and Mobile Robots. The International Journal of Robotics Research, 1986, 5(1), 90-98. https://doi. org/10.117r/027836498600500106

17. Kim, S. Kwon, S. J. Nonlinear Optimal Control Design for Underactuated Two-Wheeled Inverted Pendulum Mobile Platform. IEEE/ASME Transactions on Mechatronics, 2017, 22(6), 2803-2808. https://doi. org/10.1109/TMECH.2017.2767085

18. Lee, J., Mukherjee, R., Khalil, H. K. Output Feedback Stabilization of Inverted Pendulum on a Cart in the Presence of Uncertainties. Automatica, 2015, 54, 146 157. https://doi.org/10.1016/j.automatica.2015.01.013

19. Li, Y., Yuan, D. L. Principles of Automatic Control. Northwestern Polytechnic University Press, Xi'an, 2015.

20. Li, Z. J. Yang, C. G. Neural-Adaptive Output Feedback Control of a Class of Transportation Vehicles Based on Wheeled Inverted Pendulum Models. IEEE Transactions on Control Systems Technology, 2012,20(6), 15831591. https://doi.org/10.1109/TCST.2011.2168224

21. Li, Z. J., Yang, C. G., Su, C. Y., Deng, J., Zhang, W. D. Vision-Based Model Predictive Control for Steering of a Nonholonomic Mobile Robot. IEEE Transactions on Control Systems Technology, 2016, 24(2), 553-564.

22. Liang, D. K., Sun, N., Wu, Y. M., Fang, Y. C. Trajectory Planning-Based Control of Underactuated Wheeled Inverted Pendulum Robots. Science China Information Science, 2019, 62(5), 050207. https://doi.org/10.1007/ s11432-018-9623-3

23. Mabrouk, M. H., McInnes, C. R. Solving the Potential Field Local Minimum Problem Using Internal Agent States. Robotics and Autonomous Systems, 2008, 56(12), 1050-1060. https://doi.org/10.1016/j.robot.2008.09.006

24. Macktoobian, M., Shoorehdeli, M. A. Time-Variant Artificial Potential Field (TAPF): a Breakthrough in Power-Optimized Motion Planning of Autonomous Space Mobile Robots. Robotica, 2016, 34(5), 1128-1150. https://doi.org/10.1017/S0263574714002100

25. Malone, N., Chiang, H. T., Lesser, K., Oishi, M., Tapia, L. Hybrid Dynamic Moving Obstacle Avoidance Using a 
Stochastic Reachable Set-Based Potential Field. IEEE Transactions on Robotics , 2017, 33(5), 1124-1138. https://doi.org/10.1109/TRO.2017.2705034

26. Montiel, O., Orozco-Rosas, U., Sepulveda, R. Path Planning for Mobile Robots Using Bacterial Potential Field for Avoiding Static and Dynamic Obstacles. Expert Systems with Applications, 2015, 42(12), 51777-5191. https://doi.org/10.1016/j.eswa.2015.02.033

27. Ning, Y. G., Yue, M., Yang, L., Hou, X. Q. A Trajectory Planning and tracking Control Approach for Obstacle Avoidance of Wheeled Inverted Pendulum Vehicles. International Journal of Control, 2020, 93(7), 1735-1744. https://doi.org/10.1080/00207179.2018.1530455

28. Ogren, P., Leonard, N. E. A Convergent Dynamic Window Approach to Obstacle Avoidance. IEEE Transactions on Robotics, 2005, 21(2), 188-195. https://doi. org/10.1109/TRO.2004.838008

29. Peng, Y. W., Liu, J. K., He, W. Boundary Control for a Flexible Inverted Pendulum System Based on a PDE Model. Asian Journal of Control, 2018, 20(1), 12-21. https://doi.org/10.1002/asjc.1336

30. Sun, J. Y., Tang, J., Lao, S. Y. Collision Avoidance for Cooperative UAVs with Optimized Artificial Potential Field Algorithm. IEEE Access, 2017, 5, 18382-18390. https://doi.org/10.1109/ACCESS.2017.2746752

31. Wang, L.X. A Course in Fuzzy Systems and Control. Prentice-Hall, Englewood Cliffs, 1997.

32. Yang, C. G., Ju, Z. J., Liu, X. F., Zhong, J. P., Andy, A. Control Design for Systems Operating in Complex Environments. Complexity, 2019, 6723153. https://doi. org/10.1155/2019/6723153

33. Yang, C. G., Li, Z. J., Li, J. Trajectory Planning and Optimized Adaptive Control for a Class of Wheeled Inverted Pendulum Vehicle Models. IEEE Transactions on Cybernetics, 2013, 43(1), 24-36. https://doi.org/10.1109/ TSMCB.2012.2198813
34. Ye, J. Hybrid Trigonometric Compound Function Neural Networks for Tracking Control of a Nonholonomic Mobile Robot. Intelligent Service Robotics, 2014, 7(4), 235-244. https://doi.org/10.1007/s11370-014-0155-9

35. Yokoyama, K. Takahashi, M. Dynamics-Based Nonlinear Acceleration Control with Energy Shaping for a Mobile Inverted Pendulum with a Slider Mechanism. IEEE Transactions on Control Systems Technology, 2016, 24(1), 40-55. https://doi.org/10.1109/ TCST.2015.2417499

36. Yue, M., An, C., Sun, J. Z. An Efficient Model Predictive Control for Trajectory Tracking of Wheeled Inverted Pendulum Vehicles with Various Physical Constraints. International Journal of Control, Automation and Systems, 2018, 18(1), 265-274. https://doi.org/10.1007/ s12555-016-0393-z

37. Yue, M., Hou, X. Q., Gao, J. J., Yang, L. RBFNN-Based Identification and Compensation Mechanism for Disturbance-Like Parametric Friction with Application to Tractor-Trailer Vehicles. Asian Journal of Control, 2019, 21(6), 1-13. https://doi.org/10.1002/asjc.1884

38. Yue, M., Ning, Y. G., Zhao, X. D., Zong, G. D. Point Stabilization Control Method for WIP Vehicles Based on Motion Planning. IEEE Transactions on Industrial Informatics, 2019, 15(6), 3368-3378. https://doi.org/10.1109/ TII.2018.2875048

39. Zare K., Mardani, M. M., Vafamand, N., Khooban, M. H., Sadr, S. S., Dragicevic, T. Fuzzy-Logic-Based Adaptive Proportional-Integral Sliding Mode Control for Active Suspension Vehicle Systems: Kalman Filtering Approach. Information Technology and Control, 2019, 48(4), 648-659.

40. Zhang, X. B., Wang, J. R., Fang, Y. C., Yuan, J. Multilevel Humanlike Motion Planning for Mobile Robots in Complex Indoor Environments. IEEE Transactions on Automation Science and Engineering, 2019, 16(3), 12441258. https://doi.org/10.1109/TASE.2018.2880245 